\title{
Incremental Role of Fluorine 18-Fluorodeoxyglucose Positron Emission Tomography/Computed Tomography in the Assessment of Computed Tomography-Inconspicuous Pancreatic Lesions
}

\author{
Fathinul Fikri Ahmad Saad, ${ }^{1}$ Anna Misyail Abdul Rashid, ${ }^{2}$ and Mohamad Syafeeq Faeez Md Noh ${ }^{3, *}$
}

\begin{abstract}
Background: Pancreatic malignancies encompass a heterogenous group of disorders, with poor prognosis at diagnosis. Traditionally, conventional computed tomography (CT) has been used for diagnosis, staging, and follow up. However, this technique lacks functional information; and is limited in diagnosis of occult pancreatic disease. Hybrid imaging in the form of positron emission tomography (PET)/CT provides a potential avenue for early detection and subsequent appropriate therapy.

Case Presentation: A 60-year-old male, with a history of abdominal aortic aneurysm which was repaired, came with a complaint of 2 months history of back pain, radiating to the front. The pain was relieved on leaning forward, and aggravated by lying on his back. CT angiography of the abdomen was done, which revealed a concealed aortic aneurysm and a significant atrophy of the pancreatic tail. The serum cancer antigen (CA) 19-9 was elevated $(50.0 \mathrm{U} / \mathrm{mL}$, reference range $0.0-37.0 \mathrm{U} / \mathrm{mL})$. At this juncture, the PET scan done revealed no discernible abnormalities. Patient was put on close follow-up in view of the rising trend of CA 19-9 levels. Three months following the initial scans, a repeat ${ }^{18} \mathrm{~F}$-FDG (fluorine 18 fluorodeoxyglucose) PET/CT revealed an FDG-avid lesion at the neck of the pancreas on PET without perceptible changes on the correlated $C T$. A Whipple's procedure ensued, with histopathological examination findings of pancreatic adenocarcinoma.

Conclusion: This article discusses the role of PET/CT in the early diagnosis of inconspicuous pancreatic lesions; which could have averted immediate medical therapy.
\end{abstract}

Keywords: computed tomography; pancreatic malignancy; PET/CT

\section{Introduction/Background}

In the past, computed tomography (CT) was the standard imaging method in assessment of pancreatic lesions, in addition to ultrasound. ${ }^{1-3}$ It became the preferred imaging method for obvious reasons; fast image acquisition, ability in depicting the entire organ imaged with good resolution, and multiplanar as well as postprocessing capabilities. However, it remained as a form of structural assessment. ${ }^{1-3}$ No information on the function of the or- gans could be extracted from the images acquired. In recent years, combined positron emission tomography (PET) and CT exploiting fluorodeoxyglucose (FDG) have been widely utilized as the standard of care in medical practice, particularly when either structural or functional information is inadequate to make a diagnosis. This case report documents the value of PET/CT as opposed to conventional CT alone as a potential tool for presurgical treatment planning.

${ }^{1}$ Centre for Diagnostic Nuclear Imaging, Universiti Putra Malaysia, Serdang, Malaysia.

Departments of ${ }^{2}$ Medicine and ${ }^{3}$ Imaging, Faculty of Medicine and Health Sciences, Universiti Putra Malaysia, Serdang, Malaysia.

*Address correspondence to: Mohamad Syafeeq Faeez Md Noh, MD, Department of Imaging, Level 3, Faculty of Medicine and Health Sciences, 43400 Universiti Putra Malaysia, Serdang, Malaysia, E-mail: msf.mdnoh@gmail.com

(C) Fathinul Fikri Ahmad Saad et al. 2017; Published by Mary Ann Liebert, Inc. This is an Open Access article distributed under the terms of the Creative Commons Attribution License, which permits unrestricted use, distribution, and reproduction in any medium, provided the original work is properly cited. 

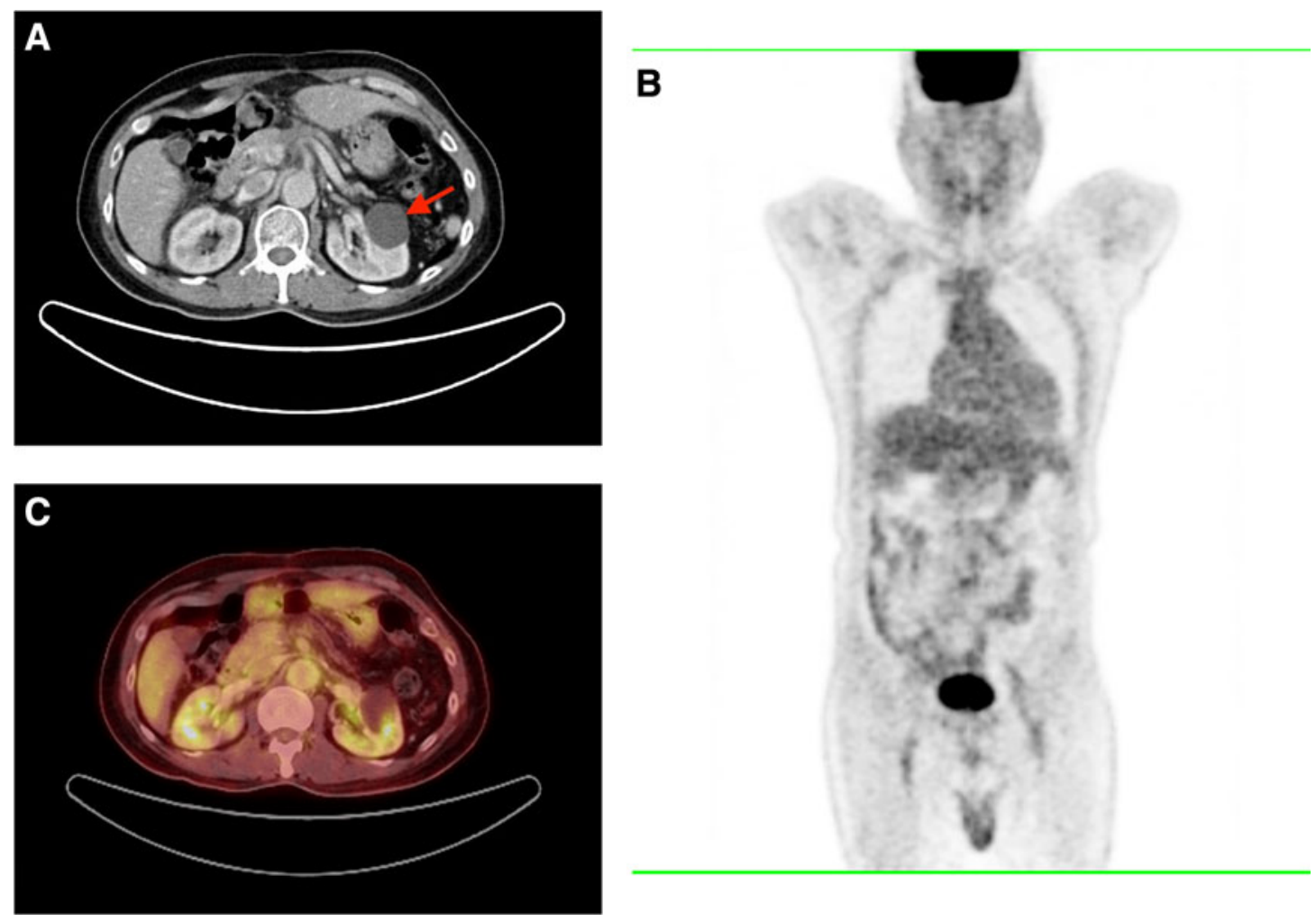

FIG. 1. Conventional $C T, P E T$, and PET/CT fusion images (pre). (A) Conventional $C T$ in axial section at the level of the pancreas showing an atrophic pancreatic tail. No discernible mass lesion present. (B) PET image in coronal section, displaying normal physiological uptake; note that no abnormal FDG-avid focus is present at the pancreatic region. (C) PET/CT fusion image (axial section), also at the level of the pancreas showing no abnormal uptake at the pancreatic region. Note the incidental finding of left simple renal cyst (arrow). CT, computed tomography; FDG, fluorodeoxyglucose; PET, positron emission tomography.

\section{Case Report/Presentation}

A previously well 60-year-old male, who had a history of aorto-biiliac grafting for an incidental finding of abdominal aortic aneurysm, came to the hospital with complaints of episodes of back pain. He reports these unprovoked episodes of back pain to be slightly relieved when leaning forward, while worsened when he lies down on his back. It was initially tolerable, only to recur more frequently. Initial blood investigations only revealed slightly elevated fasting blood glucose levels. All other blood investigations were unremarkable. In view of the previous surgery and current complaint, a CT angiography of the abdomen was done, which revealed an atrophic pancreatic tail without conspicuous mass density. There was no evidence of hepatobiliary calculous disease. A serum cancer an- tigen (CA) 19-9 was ordered, which was elevated at $50.0 \mathrm{U} / \mathrm{mL} .{ }^{18} \mathrm{~F}$ (fluorine 18)-FDG PET/CT showed no abnormal uptakes (Fig. 1). He was advised to be closely followed up; taking into account the elevated serum CA 19-9 level with no obvious imaging evidence of pathology, despite being symptomatic. After 3 months, a repeat PET/CT was done, along with another serum CA 19-9. CT showed unchanged findings from the previous scan, however, unexpectedly PET revealed a focal area of increased uptake at the neck of the pancreas (Fig. 2). Serum CA 19-9 was elevated compared with baseline, at $70.0 \mathrm{U} / \mathrm{mL}$. A possible diagnosis of pancreatic malignancy was considered. After thorough discussion with the patient, a decision was made to pursue surgical resection. Patient underwent a Whipple's procedure. Gross specimen showed a fibrotic 


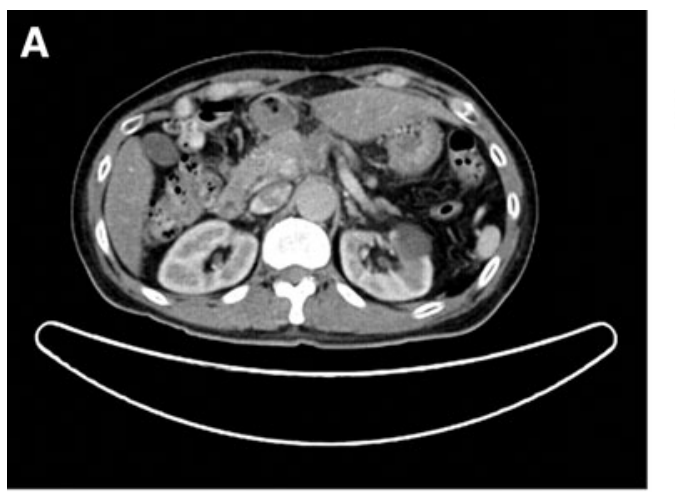

B

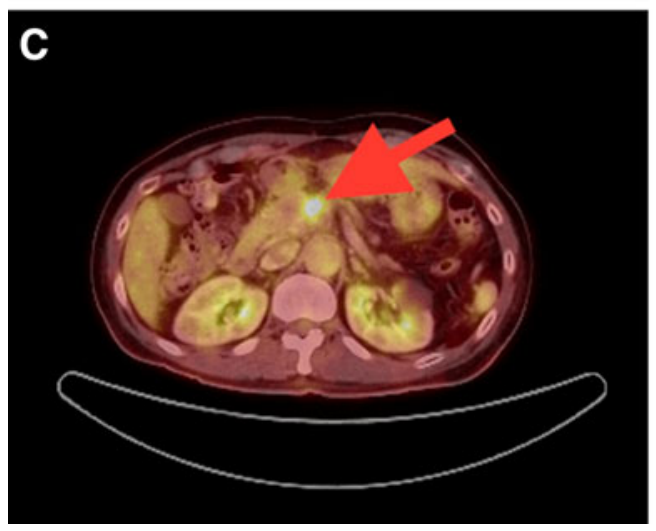

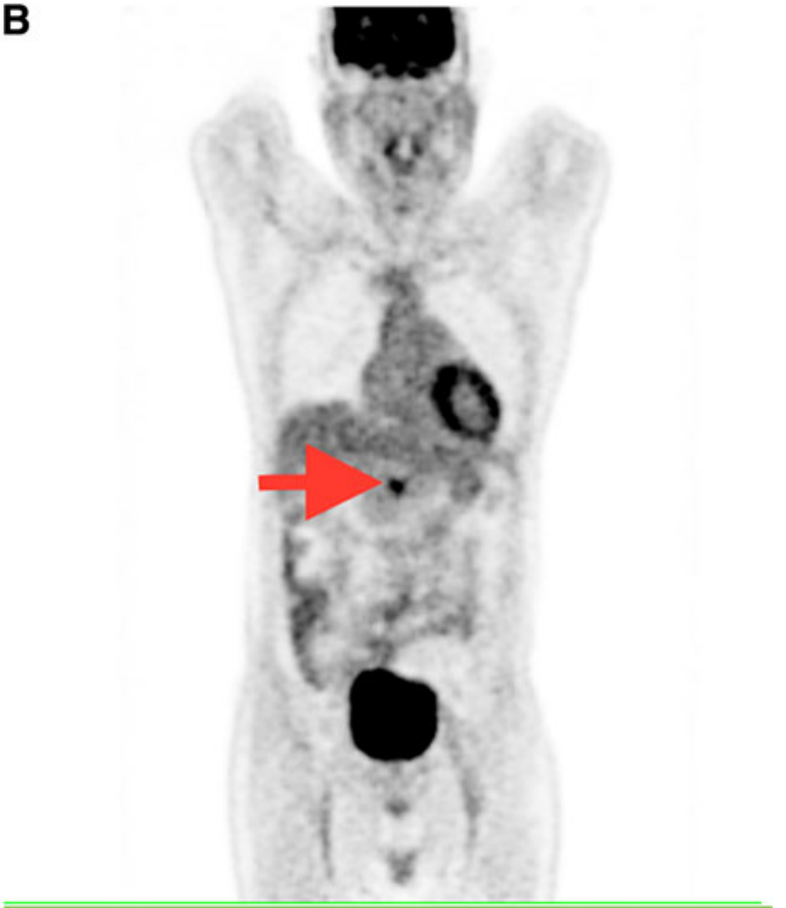

FIG. 2. Conventional CT, PET, and PET/CT fusion images (post) (A) Conventional CT in axial section at the level of the pancreas showing an atrophic pancreatic tail. No discernible mass lesion present. This is essentially similar to the previous CT study (Fig. 1A). (B) PET image in coronal section, displaying an FDG-avid focus at the region of the pancreas (arrow). Note that this was not present on previous study (Fig. 1B). (C) PET/CT fusion image (axial section), at the level of the pancreas, displaying an FDG-avid focus (arrow) corresponding to the region displayed on the PET image (B). nodule, measuring $\sim 5 \times 5 \mathrm{~mm}$ at the pancreatic neck. The peritoneal washing cytology was negative, with no extra-glandular and lymph node involvement. Complete resection with clear surgical margins was achieved, and histopathological examination confirmed adenocarcinoma of the neck of the pancreas.

\section{Discussion}

Pancreatic lesions encompass a wide group of disorders which may be benign or malignant. The importance of timely diagnosis is crucial to ensure a successful, salvageable surgical treatment. Pancreatic malignancies are known to carry a poor prognosis at the time of diagnosis. Pancreatic ductal adenocarcinoma is the fourth leading cause of cancer-related death in men and women, and the second most common cause of digestive cancer-related death. ${ }^{1,2}$

This peculiar case documents the potential value of combined PET/CT in a microscopic disease; which was not readily disclosed on CT. He was symptomatic with an unexplained elevated serum CA 19-9. The incremental value of FDG-PET in this case confirmed its usefulness in tracking early metabolic changes that underpin the cellular reprogramming and the altered glucose metabolism. Therefore, structural-based techniques, for example, CT or magnetic resonance imaging would hamper the signaling of the functional changes. ${ }^{4} \mathrm{PET}$, in combination with $\mathrm{CT}$, alerted us to the exact etiology; which turned out being an adenocarcinoma of the pancreatic neck. Taking into 
account the poor prognosis of pancreatic malignancies when eventually diagnosed, hybrid imaging serves as an early and life-saving imaging method; such as in this instance.

However, conventional imaging methods are not without value. Diehl et al. showed that the utilization of helical CT allows detection of pancreatic malignancy in $97 \%$ of cases. ${ }^{5}$ Dual phase helical CT arterial portography is shown to be superior to conventional angiography when assessing resectability of pancreatic malignancies; and that the combination of these two methods may increase diagnostic accuracy. ${ }^{6}$ Local tumor resectability is still best assessed via conventional CT; but the addition of ${ }^{18} \mathrm{~F}$ FDG PET may alter clinical management of patients suspected to have pancreatic malignancy. ${ }^{7}$

In retrospect, we postulated a few reasons that possibly contributed to the diagnosis made at 3 months following the initial PET/CT. Serum CA 19-9 is only specific in $75 \%$ of patients with pancreatic cancer, while it is raised in $10 \%$ of benign conditions. Lesions measuring less than $2 \mathrm{~cm}$ may not be readily detected by $\mathrm{CT}$, and in those which do measure more than $2 \mathrm{~cm}$, about $10 \%$ of pancreatic adenocarcinomas appear isoattenuating at contrast enhanced CT. ${ }^{1}$ Small volume lesions below PET resolution may not be identifiable. Our patient had a $5 \times 5 \mathrm{~mm}$ lesion at 3 months, which suggests that at initial scan, it may have been smaller; thus not detected on PET/CT.

The heterogeneity of pancreatic tumors, for example, neuroendocrine tumors, equates to a protracted clinical course and late presentation, in which case conventional CT may not be useful in targeting primary changes. ${ }^{8}$ In this particular case, PET/CT has been shown to be able to detect occult lesions on conventional imaging such as CT, as the hypermetabolism associated with tumors can occur before anatomical changes are evident. ${ }^{9}$ In advanced cases with metastasis, PET/CT is proved invaluable in sparing patients from unnecessary surgery. ${ }^{10}$ Multiple studies have demonstrated the sensitivity of PET/CT being higher as opposed to standard cross sectional imaging for detecting distant metastasis. ${ }^{3}$ In situations where treatment has been instituted, PET/CT also proves to be a valuable method in evaluating response, assessment of disease recurrence, and in prognostication. ${ }^{11,12}$

However, the application of PET/CT as an imaging modality in occult pancreatic malignancies is not without limitations. A study by Yasuda et al. reported an incident rate of newly diagnosed pancreatic cancer at $0.3 \% .{ }^{13}$ Some of the known attributes of FDG-negative pancreatic le- sions include neuroendocrine tumor cell lineages and small volume lesions below PET resolution, which hamper timely diagnosis. ${ }^{14}$ A recent meta-analysis reports PET as being a valuable diagnostic and predictive tool for pancreatic cancer; its value in staging remains to be proved. ${ }^{15}$ Non-FDG PET utility, for example, 68 GaDOTA NOC-a marker for somatostatin receptor tumors would complement the shortcomings of FDG PET. This is where clinical suspicion plays a central role. A high index of suspicion may render the physician to closely monitor and schedule more frequent interval imaging.

\section{Conclusion}

The role and value of hybrid imaging in the form of PET/CT is promising; providing both structural and functional information to the treating clinician. This allows portrayal of objective imaging evidence of inconspicuous pancreatic lesions, enabling institution of curative therapy. However, recognition of the potential limitations and pitfalls needs to be addressed and acknowledged in guiding treatment strategies.

\section{Informed Consent Statement}

Consent has been obtained from the patient for publication of the case details and any accompanying images.

\section{Author Disclosure Statement}

No competing financial interests exist.

\section{References}

1. Sahani DV, Bonaffini PA, Catalano OA, et al. State-of-the-art PET/CT of the pancreas: current role and emerging indications. Radiographics. 2012;32:1133-1158.

2. Tummala $P$, Junaidi $O$, Agarwal B. Imaging of pancreatic cancer: an overview. J Gastrointest Oncol. 2011;2:168-174.

3. Pietryga JA, Morgan DE. Imaging preoperatively for pancreatic adenocarcinoma. J Gastrointest Oncol. 2015;6:343-357.

4. Fathinul Fikri AS, Lau WFE. Significance of subcentimetre 18F-FDG PET/CT pulmonary abnormality in patients with known extrapulmonary malignancy. Biomed Imaging Interv J. 2010;6:e34.

5. DiehI SJ, Lehmann KJ, Sadick M, et al. Pancreatic cancer: value of dualphase helical CT in assessing resectability. Radiology. 1998;206:373378.

6. Jin Z, Li X, Cai L. Assessing the resectability of pancreatic ductal adenocarcinoma: comparison of dual-phase helical CT arterial portography with conventional angiography. Chin Med Sci J. 2001;16:40-45.

7. Rose DM, Delbeke D, Beauchamp RD, et al. ${ }^{18}$ FluorodeoxyglucosePositron Emission Tomography in the management of patients with suspected pancreatic cancer. Ann Surg. 1999;229:729-737.

8. Fathinul Fikri AS, Kroiss A, Ahmad AZF, et al. Localization and prediction of malignant potential in recurrent pheochromocytoma/paraganglioma (PCC/PGL) using 18F-FDG PET/CT. Acta Radiol. 2014;55.

9. Jha P, Bijan B. PET/CT for pancreatic malignancy: potential and pitfalls. J Nucl Med Technol. 2015;43:92-97.

10. Kim R, Prithviraj G, Kothari N, et al. PET/CT fusion scan prevents futile laparotomy in early pancreatic cancer. Clin Nucl Med. 2015;40:e501e505. 
11. Dibble EH, Karantanis D, Mercier G, et al. PET/CT of cancer patients: part 1, pancreatic neoplasms. AJR. 2012;199:952-967.

12. Farma JM, Santillan AA, Melis M, et al. PET/CT fusion scan enhances $C T$ staging in patients with pancreatic neoplasms. Ann Surg Oncol. 2008;15:2465-2471.

13. Yasuda $\mathrm{S}$, Ide $\mathrm{M}$, Fujji $\mathrm{H}$, et al. Application of positron emission tomography imaging to cancer screening. Br J Cancer. 2000;83:1607-1611.

14. Delbeke D, Martin WH. Update of PET and PET/CT for hepatobiliary and pancreatic malignancies. HPB. 2005;7:166-179.

15. Wang Z, Chen JQ, Liu JL, et al. FDG-PET in diagnosis, staging and prognosis of pancreatic carcinoma: a meta-analysis. World J Gastroenterol. 2013;19:4808-4817.
Cite this article as: Ahmad Saad FF, Abdul Rashid AM, Md Noh MSF (2017) Incremental role of fluorine 18-fluorodeoxyglucose positron emission tomography/computed tomography in the assessment of computed tomography-inconspicuous pancreatic lesions, Journal of Pancreatic Cancer 3:1, 66-70, DOI: 10.1089/pancan.2017.0014.

$\begin{aligned} & \text { Abbreviations Used } \\ &{ }^{18} \mathrm{~F}= \text { fluorine } 18 \\ & 68 \mathrm{Ga}-\mathrm{DOTA} \mathrm{NOC}= 68 \mathrm{Ga} \text {-labeled }[1,4,7,10 \text {-tetraazacyclododecane- } \\ &1,4,7,10 \text {-tetraacetic acid }]-1-N a \mid 3-\text { octreotide } \\ & \mathrm{CA} 19-9=\text { cancer antigen } 19-9 \\ & \mathrm{CT}=\text { computed tomography } \\ & \mathrm{FDG}=\text { fluorodeoxyglucose } \\ & \mathrm{PET}=\text { positron emission tomography }\end{aligned}$

\section{Publish in Journal of Pancreatic Cancer}

- Immediate, unrestricted online access

- Rigorous peer review

- Compliance with open access mandates

- Authors retain copyright

- Highly indexed

- Targeted email marketing

\section{liebertpub.com/pancan}

